\title{
EVALUACIÓN DE LA CALIDAD DE INFORMACIÓN SOBRE EL EMBARAZO EN PÁGINAS WEB SEGÚN LAS GUÍAS PERUANAS
}

\author{
Rodrigo M. Carrillo-Larco ${ }^{1,2, a}$, José E. Pérez-Lu',b, \\ Eduardo Alvarado-Vásquez ${ }^{3, b}$, Walter H. Curioso ${ }^{3,4, c}$
}

\begin{abstract}
RESUMEN
Objetivos. El objetivo del presente trabajo es evaluar la calidad de páginas web en español, que contengan información sobre los síntomas del embarazo. Materiales y métodos. Se realizó un estudio transversal y analítico, empleando la metodología previamente validada por Curró et al. para evaluar la calidad de páginas web; estas páginas fueron recuperadas a través de búsquedas en Google, MSN.com y Yahoo.com. La evaluación de la calidad incluyó tres componentes: contenido médico, responsabilidad y usabilidad de la página web. El contenido médico fue contrastado con la información disponible en las guías nacionales de Atención Integral de la Salud Sexual y Reproductiva, del Ministerio de Salud del Perú. Resultados. Se evaluaron 99 páginas web con contenido en español. El 26,3\% presentó media adherencia al patrón de oro. En el 32,3\% se encontraron los tres factores evaluados de las guías nacionales; $37,4 \%$ eran páginas web de mala calidad. El $24,2 \%$ eran blogs los cuales estaban asociados, como factor protector, a páginas web de mala calidad (OR: 0,1, IC 95\%: 0,02-0,5). Conclusiones. La mayoría de páginas web evaluadas en español, relacionadas con síntomas del embarazo, son de mala calidad. Estos resultados indican que se requiere educar adecuadamente a los pacientes y al público en general así como evaluar la calidad de información que se encuentra en Internet.
\end{abstract}

Palabra clave: Acceso a la información; Internet; Evaluación; Educación en Salud; Embarazo (fuente: DeCS BIREME).

\section{EVALUATION OF THE QUALITY OF INFORMATION ABOUT PREGNANCY FOUND IN WEBPAGES ACCORDING TO THE PERUVIAN GUIDELINES}

\begin{abstract}
Objectives. The objective of this study is to evaluate the information quality about pregnancy symptoms in Spanish available in the internet. Materials and methods. This is an analytic, cross-sectional study. In order to evaluate the selected websites, we used a methodology previously validated by Curró et al. The websites were selected from Google, MSN.com and Yahoo.com. The quality evaluation included three components: medical contents, accountability and usability of the website. The medical content was compared with the data available in the national guidelines for Comprehensive Sexual and Reproductive Health, from the Peruvian Ministry of Health. Results. Ninety nine websites in Spanish were evaluated. From them, $26.3 \%$ had medium adherence to the gold standard document. In $37.4 \%$ the websites were of low quality. A total of $24.2 \%$ were blogs, they were associated (as a protrective factor) to sites of poor quality (OR: $0.1,95 \% \mathrm{Cl}: 0.02$ to 0.5). Conclusions. The majority of websites in Spanish related to pregnancy symptoms have low quality. These findings indicate the need to properly educate patients and general public, and a critical evaluation of the information quality found at the Internet as well.
\end{abstract}

Key words: Access to information; Internet; Evaluation; Health Education; Pregnancy (source: MeSH NLM).

\section{INTRODUCCIÓN}

La cantidad de información médica que se encuentra en Internet crece constantemente ${ }^{(1-3)}$, supera a la televisión y a otros medios de comunicación en lo que respecta a la difusión y disponibilidad de ese tipo de información (4). Sin embargo, la información en Internet proviene de una gran cantidad de fuentes que no siempre son correctas, por lo que es difícil para el público en general, discernir cuáles son las páginas más indicadas para encontrar información relacionada con su salud ${ }^{(3,5,6)}$. Asimismo, es posible que la información encontrada por el paciente influya en su propio tratamiento o el de otro familiar, además de intervenir en la relación con el médico ${ }^{(1,7)}$.

Los médicos, o aquellos profesionales de la salud que emplean Internet como medio para acercarse a los pacientes, deben aplicar los principios éticos que siguen en la vida real; por ejemplo, el compromiso de servir y ayudar, sobre el deseo de beneficios económicos (8).

\footnotetext{
Facultad de Medicina "Alberto Hurtado", Universidad Peruana Cayetano Heredia. Lima, Perú.

Sociedad Científica de Estudiantes de Medicina Cayetano Heredia (SOCEMCH). Lima, Perú. Facultad de Salud Pública y Administración, Universidad Peruana Cayetano Heredia. Lima, Perú.

Oficina General de Estadística e Informática (OGEI), Ministerio de Salud. Lima, Perú.

Estudiante de Medicina; ${ }^{\mathrm{b}}$ médico cirujano; ${ }^{\mathrm{c}}$ médico, magíster en Salud Pública, especialista en Informática Biomédica.

* Los resultados preliminares del estudio han sido presentados en el libro de resúmenes del XXV Congreso Científico Nacional de Medicina SOCIMEP, Cusco, Perú. Agosto 2011.
}

Recibido: 04-10-11 Aprobado: 11-01-12 
Lamentablemente, el conocimiento, las capacidades y los usos, con frecuencia se desarrollan más rápido que los lineamientos o principios bajo los cuales se deberían guiar quienes desarrollan una nueva área, en este caso la información médica de libre acceso en Internet ${ }^{(8,9)}$. Así pues, el principal inconveniente de Internet es su naturaleza anárquica, pues promueve el libre debate y la libre publicación, lo cual puede traer problemas de calidad (10)

Muchas personas difunden información en las páginas web sin tener entrenamiento ni dominio del tema sobre el cual opinan o comentan, a las cuales el público en general tiene acceso (11); y aun cuando las páginas web parezcan confiables, el contenido no siempre lo es; muchas veces no es lo suficientemente claro para el público objetivo ${ }^{(12,13)}$. Evaluaciones sobre la calidad de la información en Internet, en el contexto de salud sexual y reproductiva, han demostrado que en temas como el aborto, la calidad de la información es baja (2). Latthe et al. evaluaron doce páginas sobre esterilización femenina y encontraron que ninguna de las páginas cumplía con todos los requisitos de calidad ${ }^{(3)}$. Por otra parte, Thompson y Graydon encontraron que menos del $30 \%$ de páginas con información relacionada al Metrotexate y los efectos que este puede ocasionar son consideradas confiables ${ }^{(14)}$. En general, filtrar la información de calidad en Internet, consume tiempo y es una actividad complicada ${ }^{(15)}$.

Por ello es necesario investigar la calidad de páginas web con contenido médico; sin embargo, son pocos los estudios en español que lo han hecho (16-18). Además, es importante poner a disposición de toda la población, información sobre fuentes confiables, pues el consumo de Internet ha crecido en los últimos años y no es exclusivo de un nivel socioeconómico ${ }^{(19)}$. Un ejemplo concreto del acceso a Internet en todos los estratos sociales son las cabinas públicas, las cuales son muy frecuentadas por público de diferentes edades y diferentes estratos ${ }^{(19)}$. El público busca información sobre temas de salud y, se ha visto que entrenar en cómo seleccionar la información más conveniente, da resultados importantes (20). Por otra parte, no solo se deben considerar los aspectos extrínsecos de la información en Internet, sino también los inherentes a quienes son responsables de publicar dicha información.

Los aspectos éticos son fundamentales en la relación médico-paciente, pero también se deben considerar cuando se discute sobre información médica en Internet ${ }^{(8)}$. Los códigos de conducta o códigos de ética que deben guardar los proveedores y consumidores de salud en Internet deben ser claros y equitativos para ambas partes. Esto no es un concepto nuevo, ya que desde 1996 se vienen desarrollando códigos de conducta para el tratamiento de la información médica en Internet y el comportamiento de los proveedores y usuarios ${ }^{(9)}$.
Por todo lo descrito, la evaluación de la calidad de la información médica disponible en Internet, es un tema relevante para investigar en salud pública. El objetivo de este trabajo evaluar la calidad de páginas web en español que contengan información sobre los síntomas del embarazo, comparando la información con guías peruanas.

\section{MATERIALES Y MÉTODOS}

El presente es un estudio transversal y analítico, donde se analizaron las páginas web con información en español sobre síntomas del embarazo. Las páginas fueron ubicada en tres buscadores distintos: Google (español), MSN.com y Yahoo.com, seleccionadas por ser los más populares en el Perú (21). Asimismo, se escogió el tópico de síntomas del embarazo, pues, en el Perú, durante el periodo 2004-2010 (22) fue el tema más buscado sobre embarazo en Internet, según estadísticas de Google Insigths.

Para determinar el número mínimo de páginas por revisar, se calculó un tamaño de muestra donde se consideró que la prevalencia de páginas web de buena calidad era del $50 \%$, con un nivel de confianza del $95 \%$ y una precisión del $10 \%$. El tamaño muestral fue 99, que fue dividido entre los tres buscadores, realizando una selección de las páginas por conveniencia.

En cada uno de los buscadores se usó la siguiente fórmula de búsqueda: embarazo OR gestación "síntomas". Se excluyeron a las páginas web que: tenían como única finalidad el comercio; aquellas cuyo objetivo era motivar o ser el medio a través del cual los visitantes pueden adquirir un producto mediante una transacción monetaria y las que contenían información histórica o requerían de un pago o registro para acceder a la información. Las páginas con contenido exclusivamente comercial podían contener un sesgo que beneficie únicamente a un producto o marca en particular.

De cada buscador se seleccionaron los primeros 33 resultados, cifra similar, e incluso superior, a otros estudios publicados que evalúan páginas web en idiomas extranjeros ${ }^{(1,3)}$. En el caso de que una página ya haya sido recuperada con un buscador y se repita en otro, o si la página cumplía algún criterios de exclusión, era omitida y se ingresaba a la siguiente página en estricto orden correlativo.

En la primera etapa de evaluación, la calidad de las páginas web fue evaluada con los criterios previamente validados por Curró et al. ${ }^{(23)}$. Los resultados de la evaluación con dichos criterios son muy similares a los obtenidos con otros patrones de evaluación; sin embargo, los de Curró son aplicados a personas sin entrenamiento médico. 
Basados en los criterios de Curró, la evaluación de la calidad de las páginas web incluyó tres dimensiones o componentes: 1) contenido médico (adherencia al patrón de oro, cantidad de factores contemplados en el patrón de oro, referencias y actualización); 2) responsabilidad de autoría (nombre del autor, información sobre el autor, patrocinio certificación y si la página pertenece a una organización sin fines de lucro) y, 3) usability (usabilidad), es decir la capacidad de una interfaz de ser comprendida, ser usada y ser atractiva para un usuario (correo para comunicarse con el autor, estética, facilidad de uso e hipervínculos).

Los tres componentes expuestos fueron propuestos en el instrumento empleado para evaluar la calidad de las páginas web. Para evaluar el contenido médico se realizó una lista de verificación de todos los síntomas de embarazo y cantidad de factores que deberían de contener las páginas web según el patrón de oro. Según la evaluación de Curró, cada componente tiene como calificación una puntuación del 0 al 1 y el puntaje final de la página web es el promedio de los tres componentes. Finalmente, se categorizó la calidad de las páginas web como buena (0,67 a 1 puntos), regular $(0,33$ a 0,66 puntos) y mala (menor de 0,33 puntos).

El patrón de oro utilizado para cumplir con los criterios de evaluación de Curró et al. ${ }^{(23)}$, fueron las Guías nacionales de atención integral de la salud sexual y reproductiva, del Ministerio de Salud del Perú ${ }^{(24)}$. Este documento está dividido en siete módulos: atención integral de la salud reproductiva, atención obstétrica, manejo de emergencias obstétricas, entre otros. Se tomó la información relativa a los síntomas del embarazo, la cual fue contrastada con aquella que se encontró en las páginas web.

Laevaluación dela calidad se realizó independientemente por tres investigadores con entrenamiento y experiencia en búsqueda y evaluación de páginas web. Posteriormente, los resultados fueron contrastados y las discrepancias se resolvieron por consenso.

La segunda etapa de la evaluación se realizó con la finalidad de alcanzar los objetivos exploratorios. Se buscó la asociación a páginas web de mala calidad con las siguientes variables: cantidad de referencias, país de procedencia, institución que promueve la página, si la página es administrada/supervisada por un profesional de la salud y si la página es un blog.

Se utilizaron frecuencias para describir las variables; además, se aplicó el modelo de regresión logística para calcular el OR y poder establecer qué variables se asocian con páginas de mala calidad. Se estableció un nivel de significancia del 95\% $(p<0,05)$.

El protocolo de la investigación fue aprobado por el Comité Institucional de Ética de la Universidad Peruana Cayetano Heredia.

\section{RESULTADOS}

En total, se incluyeron 99 páginas web en el análisis. El coeficiente Kappa que hubo entre los autores durante la evaluación de cada una de las páginas web, fue 0,75 ; este valor representa una buena concordancia.

El promedio de puntaje de las páginas web fue de 0,36 $\pm 0,09$, de las cuales el $37,4 \%$ fueron de mala calidad; $62,6 \%$ de regular calidad y ninguna de buena calidad.

Del total de las páginas evaluadas, el $24,2 \%$ eran un blog. Las características incluidas dentro de los componentes de calidad, se detallan en la Tabla 1.

La estética es otra característica evaluada dentro del componente de usabilidad. La mayoría de las páginas web tuvo una estética regular $(56,6 \%)$; el $29,2 \%$ tuvo buena estética y solo $11(11,1 \%)$ fue considerada como de muy buena estética.

Con relación a las otras características evaluadas, se vio que el $90,9 \%$ de la información citada en las páginas

Tabla 1. Indicadores de los componentes de la evaluación de la calidad de las páginas incluidas en el estudio $\left(N .^{\circ}=99\right)$.

\begin{tabular}{|c|c|}
\hline & N. ${ }^{\circ}(\%)$ \\
\hline \multicolumn{2}{|l|}{ Contenido médico } \\
\hline \multicolumn{2}{|l|}{$\begin{array}{l}\text { Adherencia a la información del Patrón } \\
\text { de Oro }\end{array}$} \\
\hline No adherencia o contiene errores & $21(21,2)$ \\
\hline Media-poca adherencia & $41(41,4)$ \\
\hline Media adherencia & $26(26,3)$ \\
\hline Media-alta adherencia & $7(7,1)$ \\
\hline Adherencia completa & $3(3,0)$ \\
\hline \multicolumn{2}{|l|}{$\begin{array}{l}\text { Contempla la totalidad de componentes } \\
\text { del Patrón de Oro }\end{array}$} \\
\hline Ningún componente & $2(2,0)$ \\
\hline Un componente & $36(36,4)$ \\
\hline Dos factores & $29(29,3)$ \\
\hline Tres componentes & $32(32,3)$ \\
\hline Cita referencias pertinentes & $10(10,1)$ \\
\hline $\begin{array}{l}\text { Indica fecha de creación o de última } \\
\text { actualización }\end{array}$ & $43(43,4)$ \\
\hline \multicolumn{2}{|l|}{ Responsabilidad } \\
\hline Indica nombre del autor & $40(40,4)$ \\
\hline Tiene patrocinio comercial & $79(79,8)$ \\
\hline Indica información del autor & $12(12,1)$ \\
\hline Páginas acreditadas & $5(5,1)$ \\
\hline $\begin{array}{l}\text { Página no pertenece a organizaciones sin } \\
\text { fines de lucro }\end{array}$ & $99(100,0)$ \\
\hline \multicolumn{2}{|l|}{ Usabilidad } \\
\hline $\begin{array}{l}\text { Brinda correo y otro medio para contactar } \\
\text { al autor }\end{array}$ & $58(58,6)$ \\
\hline \multicolumn{2}{|l|}{ Facilidad de uso } \\
\hline Malo & $1(1,0)$ \\
\hline Regular & $18(18,2)$ \\
\hline Bueno & $80(80,8)$ \\
\hline \multicolumn{2}{|l|}{ Cantidad de hipervínculos } \\
\hline Malo & $1(1,0)$ \\
\hline Regular & $17(17,2)$ \\
\hline Bueno & $81(81,8)$ \\
\hline
\end{tabular}


web no tiene referencias, mientras que el $9,1 \%$ sí las especifica. La mayoría de las páginas incluidas en el estudio fueron extranjeras $(63,6 \%)$ pero el $31,3 \%$ no refiere país de procedencia. Por otra parte, cuando se evaluó qué institución promueve la página web, se encontró que el $73,7 \%$ de los casos se trataba de una empresa o institución privada; el $4,0 \%$ por una universidad, ONG, instituto o biblioteca; y el $22,2 \%$ no lo especifica. La mayoría de las páginas web $(41,4 \%)$ evaluadas no deja patente quién tiene la dirección, es decir si es dirigida o supervisada por un profesional de la salud; en este sentido solo el $26,3 \%$ estaba dirigida por un profesional de la salud y el $32,3 \%$ por una persona ajena a las ciencias de la salud.

No se encontraron asociaciones significativas entre la mala calidad de las páginas web y variables tales como la cantidad de referencias, el país de procedencia, la institución que promueve el portal o página web y tampoco si esta era dirigida por un profesional de la salud. Sin embargo, sí se encontró que la condición de blog constituye un factor protector (OR=0,1, IC 95\%: $0,02-0,47)$ contra páginas web de mala calidad. El análisis bivariado se detalla en la Tabla 2 .

\section{DISCUSIÓN}

Los resultados muestran que la mayoría de páginas web sobre síntomas del embarazo en español, son de regularmala calidad. Esto guarda concordancia con la literatura existente, ya que otras experiencias que evaluaron páginas web sobre diversos temas en salud han llegado a la misma conclusión ${ }^{(2,16,25,26)}$. En cuanto a páginas web sobre salud sexual y reproductiva en inglés, menos del $20 \%$ de ellas son consideradas como aceptables ${ }^{(1)}$.

Tabla 2. Factores asociados a una página Web de mala calidad ( $n=99)$.

\begin{tabular}{|c|c|}
\hline & $\begin{array}{l}\text { Odds ratio } \\
\text { (IC } 95 \%)\end{array}$ \\
\hline \multicolumn{2}{|l|}{ Cantidad de referencias } \\
\hline No tiene & 1 \\
\hline Entre 1 a 4 referencias & $0,4(0,1-2,3)$ \\
\hline \multicolumn{2}{|l|}{ País de procedencia } \\
\hline Extranjera & 1 \\
\hline Peruana & $7,5(0,8-70,9)$ \\
\hline No refiere & $1,0(0,4-2,5)$ \\
\hline \multicolumn{2}{|l|}{$\begin{array}{l}\text { Institución que promueve la página } \\
\text { Web }\end{array}$} \\
\hline Universidad/ONG/Instituto/Biblioteca & 1 \\
\hline $\begin{array}{l}\text { Página de la empresa/Institución } \\
\text { privada }\end{array}$ & $0,4(0,1-3,3)$ \\
\hline No refiere & $1,4(0,2-12,2)$ \\
\hline \multicolumn{2}{|l|}{ Dirección de la página Web } \\
\hline No profesional de salud & 1 \\
\hline Profesional de salud & $1,2(0,4-3,5)$ \\
\hline No refiere & $1,7(0,7-4,5)$ \\
\hline La página es un blog & $0,1(0,02-0,5)$ \\
\hline
\end{tabular}

IC $95 \%$ : intervalo de confianza al $95 \%$.
El presente estudio ha demostrado que la muestra evaluada guarda media-poca adherencia al patrón de oro. Lo mismo se determinó en otra investigación que evaluó páginas web sobre cáncer, donde se sostiene que aquella diferencia puede causar confusión en el paciente ${ }^{(11)}$. No obstante, vale mencionar que el patrón de oro fueron las guías peruanas y que solo el $5,1 \%$ de las web evaluadas fueron páginas peruanas, esto podría explicar la poca adherencia a dicho patrón. Uno de los factores que puede afectar estos resultados, es que las guías del Ministerio de Salud del Perú no incluyen algunos síntomas típicos del embarazo (como retraso menstrual o aumento de volumen mamario) y que son mencionados por muchas páginas web.

El hecho que solo algunas web incluyan referencias, es un aspecto que llama la atención, ya que es un indicador que se relaciona con la calidad. Sin embargo, dicha característica se encontró en un estudio de páginas web en inglés y en persa, donde el $76 \%$ de la muestra no presentaba referencias relevantes de la información que ofrecían (1).

Otra investigación que evaluó la calidad de las páginas web sobre escoliosis, encontró que solo el 18\% de dichas páginas estaban dirigidas por médicos ${ }^{(27)}$. En nuestro caso, el $26 \%$ estaban dirigidas por un profesional de la salud.

Por otro lado, se observó en este estudio que la mayoría de páginas web evaluadas ofreció una buena cantidad de hipervínculos, lo que también ha sido visto en otras investigaciones que han evaluado la calidad de páginas web en español ${ }^{(17)}$.

Con relación al tiempo y vigencia de la información, se encontró que no todas publican la última fecha de actualización; similar a lo encontrado en una investigación sobre páginas web con contenido sobre métodos anticonceptivos, en las que el $41,6 \%$ presentaba información desactualizada ${ }^{(28)}$. Ello puede deberse a que es frecuente que se publique una página web que luego de un tiempo, el o los autores la olvidan y dejan de actualizar el contenido. Una suerte diferente corren los Blogs, cuyos autores tienden a prestarles mayor dedicación.

La mayoría de las páginas web evaluadas son de media-baja calidad, por lo que el público en general puede tener dificultades para encontrar webs en español sobre salud, ello mismo ha sido mostrado en otras investigaciones ${ }^{(17)}$.

Los resultados aquí presentados son un llamado de atención para que se desarrollen páginas web o portales con información adecuada, relevante y de fácil acceso a la población en general. Asimismo, este estudio resalta la importancia de educar adecuadamente a los pacientes y público en general, para que evalúen la calidad de información que se encuentra en Internet; así 
sabrá cómo filtrar la información para optar por aquella que le será beneficiosa ${ }^{(10)}$.

En lo referente a la ética y, en especial a la ética médica en Internet, se aprecia que ella puede variar según la perspectiva de un profesional de la salud, de otro profesional o desde la de una persona sin profesión; además, su regulación no será la misma de una país a otro y dependerá de las experiencias previas de cada individuo ${ }^{(8)}$. De igual manera, es importante que las personas, naturales y jurídicas, propietarias de páginas web que versen sobre temas de salud, estén conscientes del poder que tienen y lo usen con responsabilidad. El número de pacientes que navegan en Internet no ha pasado desapercibido por las empresas de los rubros afines ${ }^{(8)}$; ellos, por ejemplo, podrían especificar claramente el grupo objetivo al cual va dirigida la información presentada en su página en Internet (10). De esta manera, se pueden desarrollar nuevos códigos de conducta que establezcan nuevos límites; como cuáles son los aspectos éticos de la conducta de un médico que se deben esperar en Internet, ¿se puede adaptar los ya existentes o se deben implementar nuevos? El profesionalismo del médico, de otros profesionales de la salud y de aquellos que publican información médica en Internet $u$ otros medios de comunicación masiva, debe estar siempre presente ya sea en la realidad o en el espacio virtual.

Un aspecto que no ha sido evaluado en el presente estudio y tampoco se ha encontrado en la revisión bibliográfica realizada por los autores, es la privacidad de la información que brindan los pacientes durante la búsqueda de información. La ética exige a los profesionales de la salud que guarden celosamente toda información sobre un paciente. De esta manera, los sitios en Internet con información médica o que proveen de asistencia médica, deben asegurar la privacidad de cualquier información y certificar que no será vendida o puesta en manos de un tercero ${ }^{(8)}$.

Una de las limitaciones del estudio radica en el hecho que la información en Internet cambia constantemente y los resultados aquí presentados constituyen la imagen de un momento. En el presente estudio no se consideró el estilo de búsqueda propio de cada individuo, tampoco el tiempo de descarga de cada portal web. Por otra parte, tampoco se controló que hubieran páginas web con exactamente la misma información, ya sea como resultado del mismo motor de búsqueda o en diferentes. Otra posible limitación es el instrumento usado que pondera ampliamente algunas características del autor (como el correo o la fecha de creación) y podría haber beneficiado a los blogs; por lo que es probable que en este estudio, el ser blog sea un factor protector contra la mala calidad de información.

Así mismo, el patrón de oro usado fueron las guías peruanas, lo cual pudo ser una limitación al evaluar información foránea. Tampoco se pudo evaluar la asociación entre los diferentes buscadores web y la calidad de las páginas web debido a que en la evaluación se eliminaron varias páginas que cumplían con los criterios de exclusión. Esto no permitiría evaluación no sesgada de los resultados. Finalmente, es posible que los resultados de la búsqueda hayan estado sesgados por el historial de búsquedas del ordenador del investigador, quien confeccionó la lista de páginas web a revisar.

Se recomienda el desarrollo de guías o intervenciones educativas que enseñen cómo evaluar o qué elementos tener en cuenta para saber si una página web determinada proporcionará información de calidad, actualizada y objetiva. Para ello, se puede recomendar a los pacientes y público en general, utilizar la nemotécnica PRUEBA (Propósito, Responsable, Utilidad de la información, Enlaces, Buscar el compromiso de confidencialidad y Actualización) propuesta por Curioso et al. que busca recordar a las personas los criterios que deben tener en cuenta al evaluar la información de salud en Internet (29). Además, es importante promover que los pacientes se involucren más en su salud y que la información que encuentren en Internet sea evaluada cuidadosamente, dentro de lo posible, consultada con el profesional. De igual forma, se recomienda evaluar la calidad de páginas web sobre otros temas de salud y diseñar nuevos métodos de evaluación para determinar la calidad de las web de las instituciones prestadoras de servicios de salud, tanto pública como privada. Por otro lado, los autores recomiendan hacer estudios similares pero que evalúen la ética de las páginas web con contenido médico.

Del presente estudio se puede concluir que la mayoría de páginas web en Español, que abordan el tema de síntomas del embarazo son de mediana-baja calidad; estos resultados constituyen un llamado de atención hacia los profesionales de la salud y el público en general para que sean más cautelosos con la información que encuentran en Internet.

\section{AGRADECIMIENTOS}

Este trabajo fue realizado parcialmente gracias al apoyo del Programa Mobile Citizen del Banco Interamericano de Desarrollo $y$, parcialmente, al apoyo del Proyecto QUIPU de la UPCH, un programa auspiciado por el Fogarty International Center/National Institutes of Health (FIC/NIH), proyecto: D43TW008438.

\section{Contribuciones de autoría}

Todos los autores participaron en la concepción y diseño del trabajo; en la redacción del manuscrito, su revisión crítica y aprobación de su versión final. Además, $\mathrm{RC}$ participó en la recolección y obtención de resultados; JE en la recolección y obtención de resultados, en el análisis e interpretación de datos; EA en la recolección y obtención de resultados, en el análisis e interpretación de datos; y WC en el análisis e interpretación de resultados.

\section{Fuentes de financiamiento}

El estudio ha sido autofinanciado por los autores. 


\section{Conflictos de interés}

Los autores declaran no tener ningún conflicto de interés.

\section{REFERENCIAS BIBLIOGRÁFICAS}

1. Rahnavardi M, Arabi MS, Ardalan G, Zamani N, Jahanbin $M$, Sohani $F$, et al. Accuracy and coverage of reproductive health information on the Internet accessed in English and Persian from Iran. J Fam Plan Reprod Health Care. 2008,34(3):153-7.

2. Hardwick JC, MacKenzie FM. Information contained in miscarriage-related websites and the predictive value of website scoring systems. Eur J Obstet Gynecol Reprod Biol. 2003;106(1):60-3.

3. Latthe PM, Latthe M, Khan KS. Quality of information on female sterilisation on the Internet. J Obstet Gynaecol. 2000;20(2):167-70.

4. Leung L. Internet Embeddedness: Links with Online Health Information Seeking, Expectancy Value/Quality of Health Information Websites, and Internet Usage Patterns. CyberPsychology \& Behavior. 2008;11(5):565-9.

5. Ilic $D$. The role of the internet on patient knowledge management, education, and decision-making. Telemed $\mathrm{J} \mathrm{E}$ Health. 2010;16(6):664-9.

6. Beaton C, Codd RJ, Holland PA, Gateley CA. Evaluation of the quality and accuracy of information regarding aromatase inhibitors available on the Internet. Breast $\mathrm{J}$. 2008;14(4):366-8.

7. Tozzi AE, Buonuomo PS, Ciofi degli Atti ML, Carloni E, Meloni M, Gamba F. Comparison of quality of internet pages on human papilomavirus immunization in Italian and in English. J Adolesc Health. 2010;46(1):83-9.

8. Dyer KA. Ethical challenges of medicine and health on the Internet: a review. J Med Internet Res. 2001;3(2):e23.

9. Wilson P. How to find the good and avoid the bad or ugly: a short guide to tools for rating quality of health information on the internet. BMJ. 2002;324(7337):598-602.

10. Eysenbach G. Towards ethical guidelines for e-health: JMIR theme issue on eHealth ethics. J Med Internet Res. 2000;2(1):e7.

11. Biermann JS, Golladay GJ, Greenfield ML, Baker LH. Evaluation of Cancer Information on the Internet. Cancer. 1999;86(3):381-90.

12. Kunst $H$, Groot D, Latthe PM, Latthe M, Khan KS. Accuracy of information on apparently credible websites: survey of five common health topics. BMJ. 2002;324(7337):581-2.

13. Stinson JN, Tucker L, Huber A, Harris H, Lin C, Cohen L, et al. Surfing for juvenile idiopathic arthritis: perspectives $\mathrm{O}$ quality and content of information on the Internet. J Rheumatol. 2009;36(8):1755-62.

14. Thompson AE, Graydon SL. Patient-oriented methotrexate information sites on the Internet: a review of completeness. accuracy, format, reliability, credibility, and readability. J Rheumatol. 2009;36(1):41-9.

15. Jadad AR, Gagliardi A. Rating Health Information on the Internet. JAMA. 1998;279.
16. Eysenbach G, Powell J, Kuss O, Sa ER. Empirical studies assessing the quality of health information for consumers on the world wide web: a systematic review. JAMA. 2002;287(20):2691-700.

17. Berland GK, Elliott MN, Morales LS, Algazy JI, Kravitz RL, Broder MS, et al. Health information on the Internet: accessibility, quality, and readability in English and Spanish. JAMA. 2001;285(20):2612-21.

18. Rancaño García I, Rodrigo Pendás JA, Villa Estébanez R, Abdelsater Fayad M, Díaz Pérez R, Alvarez García D. Evaluación de las páginas web en lengua española útiles para el médico de atención primaria. Aten Primaria. 2003;31(9):575-80.

19. Instituto Nacional de Estadística e Informática. La Tecnologías de la Información y Comunicación en los Hogares. Lima, Perú: INEI; 2010.

20. Curioso WH, Blas MM, Nodell B, Alva IE, Kurth AE. Opportunities for providing web-based interventions to prevent sexually transmitted infections in Peru. PLoS Med. 2007;4(2):e11.

21. Alexa [Internet]. Seattle, WA: The Web Information Company; c1996-2012 [actualizado el 7 de abril de 2011; citado el 12 de enero 2011]. Disponible en: http://www.alexa.com/.

22. Google Insight [Internet]. Mountain View, CA: Google; c2012 [citado el 5 de Julio 2011.] Disponible en: http://goo. $\mathrm{gl} / \mathrm{xcgj2}$.

23. Curró V, Buonuomo PS, Onesimo R, de Rose P, Vituzzi A, di Tanna GL, et al. A quality evaluation methodology of health web-sites for non-professionals. Med Inform Internet Med. 2004;29(2):95-107.

24. Ministerio de Salud. Guías Nacionales de Atención Integral de la Salud Sexual y Reproductiva. Lima, Perú: MINSA; 2004.

25. Conesa Fuentes Mdel C, Aguinaga Ontoso E, Hernández Morante JJ. An evaluation of the quality of health web pages using a validated questionnaire. Aten Primaria. 2011;43(1):33-40.

26. Coquard O, Fernandez S, Khazaal Y. Assessing the quality of French language web sites peraining to alcohol dependency. Sante Ment Que. 2008;33(2):207-24.

27. Mathur S, Shanti N, Brkaric M, Sood V, Kubeck J, Paulino C, et al. Surfing for scoliosis: the quality of information available on the Internet. Spine (Phila $\mathrm{Pa}$ 1976). 2005;30(23):2695-700.

28. Weiss E, Moore K. An Assessment of the Quality of Information Available on the Internet about the IUD and the Potential Impact on Contraceptive Choices. Contraception. 2003;68(5):359-64.

29. Curioso W, Castillo C, Soto A, Iglesias Q, Echevarria Z, Gotuzzo H. Cerrando la brecha digital en el Perú: Educando a personas viviendo con VIH/SIDA a evaluar la calidad de la información en salud en la Internet. Rev Peru Med Exp Salud Publica. 2007;24(3):280-5.

Correspondencia: Walter H. Curioso

Dirección: Av. Honorio Delgado 430, Lima 31, Perú.

Teléfono: (511) 3190028

Correo electrónico: walter.curioso@upch.pe 